\title{
A case with essential tremor refractory to medication successfully treated with deep brain stimulation
}

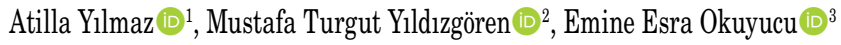 \\ ${ }^{1}$ Department of Neurosurgery, Mustafa Kemal University Medical School, Hatay, Turkey \\ ${ }^{2}$ Department of Physical Medicine and Rehabilitation, Mustafa Kemal University Medical School, Hatay, Turkey \\ ${ }^{3}$ Department of Neurology, Mustafa Kemal University Medical School, Hatay, Turkey
}

Received: November 03, 2017 Accepted: March 08, 2018 Published online: April 27, 2018

A 53-year-old, right-handed male presented at our outpatients clinic with a 15 -year history of slowly progressing essential tremor (ET) affecting his head and hands. Symptoms involved both hands, but were worse on the right-side. Postural and action tremors were found in both hands, although there was no tremor involving the lower limbs or voice. The patient did not benefit from propranolol $(80 \mathrm{mg} /$ day $)$, primidone (250 $\mathrm{mg} /$ day), and gabapentin (900 mg/day). His medical history included smoking and hypertension. The patient was referred by the neurologist to the physical medicine and rehabilitation clinic, having not any previous occupational or rehabilitation. The main complaints were hand tasks, such as writing, dressing, and eating. After conventional physical therapy (strengthening and coordination exercises were implemented five times per week for six weeks), no improvement was achieved. He was, therefore, referred to the neurosurgery clinic and underwent bilateral implantation of deep brain stimulation (DBS) (Medtronic, Minneapolis, MN, USA) into the ventralis intermedius nucleus (Vim) (Figure 1). The stimulation parameters were as follows: stimulator frequency, $130 \mathrm{~Hz}$ on both sides, voltages were 4.3 volt on the right-side and 2.6 volt on the left-side, pulse widths were 80 micro second on both sides. The
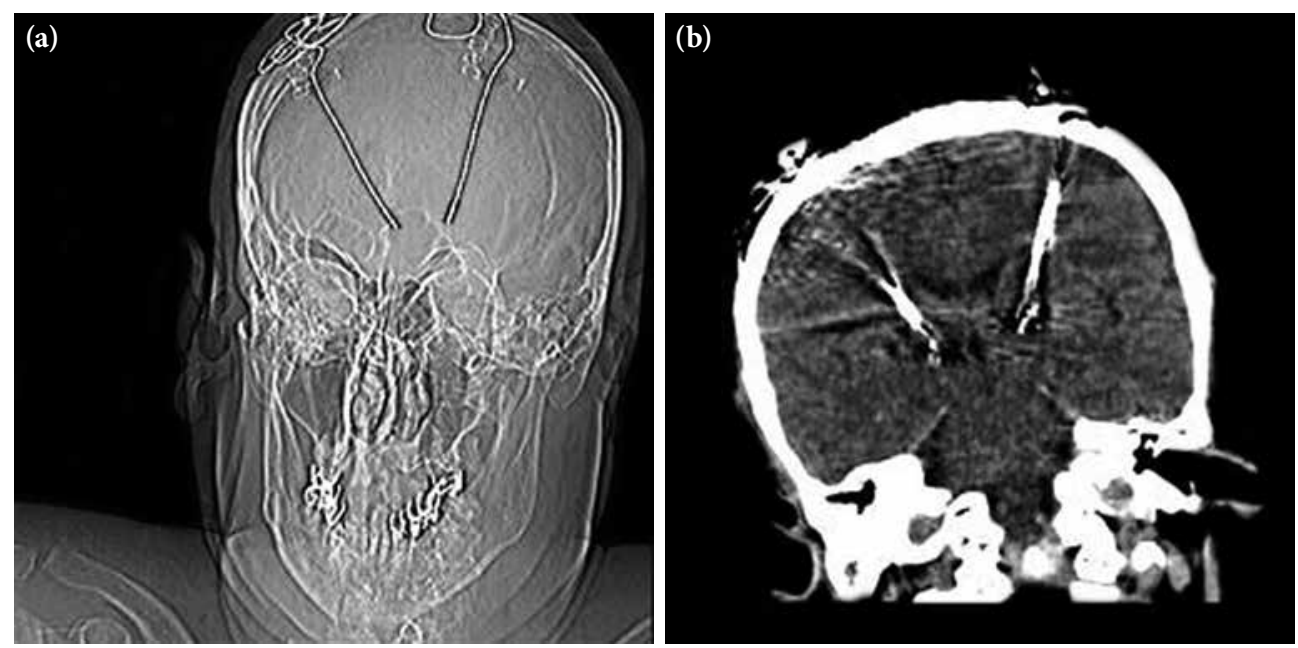

Figure 1. In the coronal view, plain radiography (a) and computed tomography scanning (b) showing that deep brain stimulation electrodes implanted in the bilateral thalamic ventral intermediate nucleus after deep brain stimulation. 


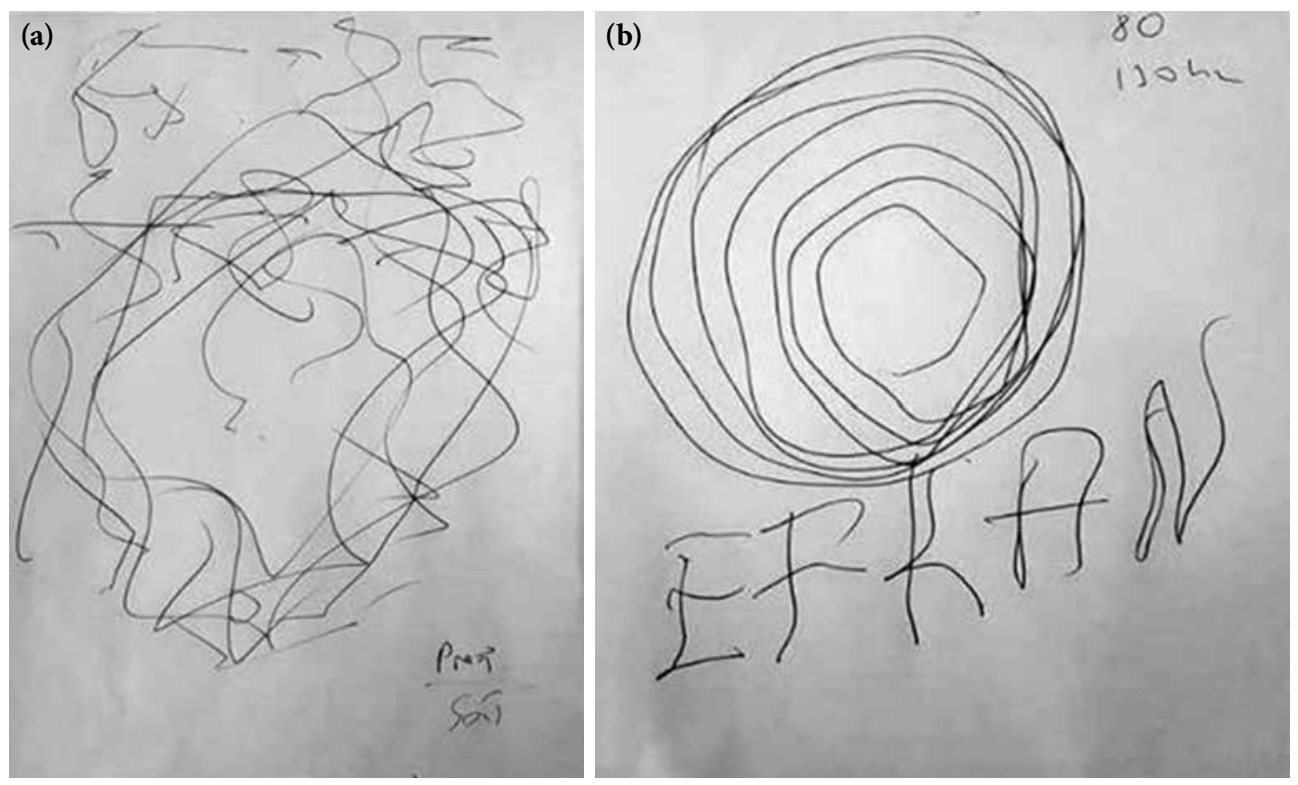

Figure 2. (a) Images showing impaired function of handwriting and hand-drawn spirals before deep brain stimulation therapy and (b) improved functions immediately after deep brain stimulation.

complaints immediately improved following the DBS. The patient was unable to write his name or hand draw spirals before DBS (Figure 2a). Writing and drawing immediately improved after DBS (Figure 2b). There were no additional problems at one month after DBS surgery.

Essential tremor, which is a progressive, ageassociated disease, is one of the most common neurological disorders, affecting up to $5 \%$ of individuals aged over 65 years. ${ }^{[1]}$ As clinical diagnosis of ET is based solely on clinical findings and neurological history, misdiagnosis of ET is common (i.e., Parkinson's disease). ${ }^{[2,3]}$ The management of patients should be determined according to the severity of tremor, the parts of the body involved, physical disability, social disability and handicap. Physical therapy treatment for ET primarily focuses on resistance training and compensatory strategies. Typically, this is applied to the upper extremity to improve strength and coordination, and to reduce tremor severity. In addition to muscle strength, behavioral relaxation therapy has been shown to decrease the tremor severity. ${ }^{[4]}$

According to the American Academy of Neurology 2011 practices, beta-blockers, most commonly propranolol, and the anticonvulsant primidone, are the first-line medications for ET (Level A). ${ }^{[5]}$ The following drugs have recommendations for treating ET: gabapentin, topiramate (Level B, probably effective); botulinum toxin, DBS, and thalamotomy (Level C, possibly effective); gamma knife surgery, pregabalin, zonisamide, and clozapine (Level $U$, insufficient evidence). In medically refractory patients, limb, head, and voice tremor may respond to botulinum toxin injections into the affected muscles. ${ }^{[5]}$ Surgical treatment such as thalamotomy or thalamic DBS for ET is reserved for selected patients with severe tremor who are unable to adequately respond to medical and physical therapy. ${ }^{[4]}$

In conclusion, while medical therapy, physical therapy and behavioral techniques may be useful in patients with mild to moderate tremor. VimDBS for ET can be performed successfully in cases refractory to these therapies or with severe tremor. Neuromodulation applications have revolutionized the approach of physicians interested in movement disorders and rehabilitation, entailing a multidisciplinary approach.

\section{Declaration of conflicting interests}

Dr. Yilmaz have disclosures with Boston Scientific and Medtronic in terms of proctorship.

\section{Funding}

The authors received no financial support for the research and/or authorship of this article.

\section{REFERENCES}

1. Louis ED, Ferreira JJ. How common is the most common adult movement disorder? Update on the worldwide prevalence of essential tremor. Mov Disord 2010;25:534-41. 
2. Louis ED. Clinical practice. Essential tremor. N Engl J Med 2001;345:887-91.

3. Abdo WF, van de Warrenburg BP, Burn DJ, Quinn NP, Bloem BR. The clinical approach to movement disorders. Nat Rev Neurol 2010;6:29-37.

4. Hedera P, Cibulčík F, Davis TL. Pharmacotherapy of essential tremor. J Cent Nerv Syst Dis 2013;5:43-55.
5. Zesiewicz TA, Elble RJ, Louis ED, Gronseth GS, Ondo WG, Dewey RB Jr, et al. Evidence-based guideline update: treatment of essential tremor: report of the Quality Standards subcommittee of the American Academy of Neurology. Neurology 2011;77:1752-5. 